Alternating Gradient Synchrotron Department

Relativistic Heavy Ion Collider Project

BROOKHAVEN NATIONAL LABORATORY

Upton, New York 11973

Spin Note

AGS/RHIC/SN No. 060

\title{
The Hall Probe Measurement and Calculation of the Half Length Helical Dipole Magnet
}

\author{
M. Okamura
}

AGS Department, Brookhaven National Laboratory, NY 11973

August 20, 1997 


\title{
The Hall Probe Measurement and Calculation of the Half Length Helical Dipole Magnet.
}

\author{
M. Okamura
}

August, 20, 1997

\begin{abstract}
In this report, fields in a Slotted type half length model magnet measured by Hall probes are compared with TOSCA 3D calculations. The measured fields agree quite well with TOSCA predictions except higher order harmonics in the body section of the helical dipole magnet. These results are showing a reliability of the $3 \mathrm{D}$ calculation by TOSCA and a stability of the fabricating method adopted in the Slotted type model.
\end{abstract}

\section{Introduction}

In middle of June, field measurements of a half length model of Slotted Type were performed. In these measurements, longitudinal field distribution was scanned by Hall probes and precise field harmonics at the center of the magnet were confirmed by rotating coils. This note is describing mainly results of the Hall probe measurement and 3D magnetic field calculations using TOSCA.

\section{Equipment for the Hall probe measurement}

Rotating coil measurements enable us to get precise field harmonics, however this method only measures averaged field surrounded by the coils. Long flat coils along the beam axis cancel the twisted magnetic fields especially in higher order multipoles. On the other hand, the Hall generators usually have a small sensing area, a few cubic $\mathrm{mm}$, and can measure local magnetic fields. Therefore we can measure field distribution in the twisted magnet without cancellation of higher order modes.

Figure 1 shows a schematic view of a head which was used for the measurement. This head was attached to a long aluminum shaft and rotated by a stepping motor. The field was sampled at 64 points per one revolution. This number is enough to estimate the sextupole and decapole components. This head was also scanned by another stepping motor to inspect the field distribution along the beam axis. In this experiment, a couple of transverse Hall generators and a three axis Hall probe were used, however the results from the three axis generator are not used in this note due to a problem with alignment.

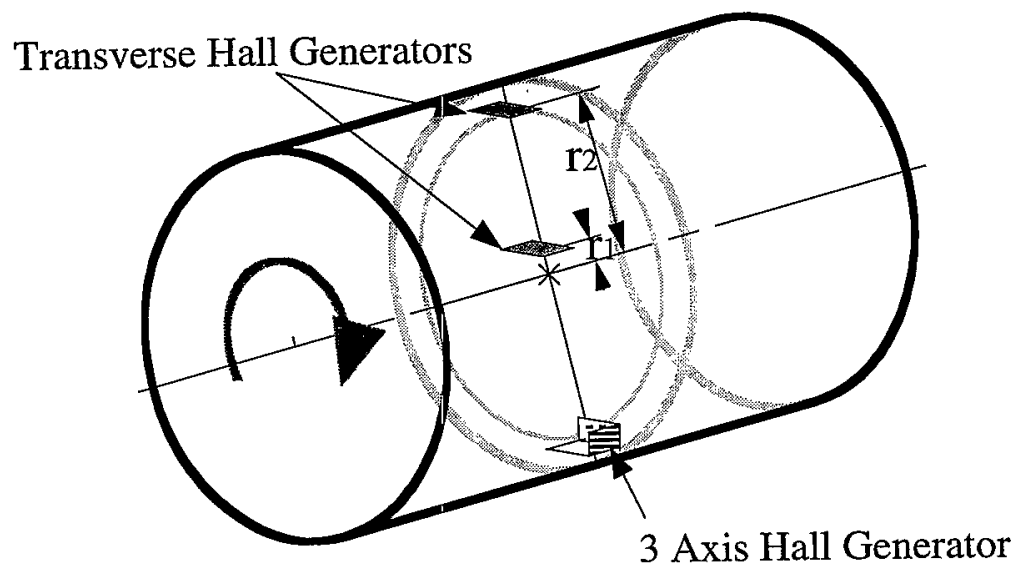

Fig. 1 Head for the Hall generators

At present, only transverse Hall generators are used in the analysis. Field each transverse Hall generators sense is expressed as 


$$
\begin{aligned}
& B_{r}=B_{0} \sum_{n=0}^{\infty} \frac{f_{n}}{k(n+1)} I_{n+1}^{\prime}((n+1) k r)\left[a_{n} \cos ((n+1) \theta)+b_{n} \sin ((n+1) \theta)\right] \cdots(1) \\
& f_{n}=\frac{2^{n+1}(n+1) !}{(n+1)^{n+1}} \frac{1}{r_{0}^{n} k^{n}} \cdots(2) .
\end{aligned}
$$

Where, $I_{n+1}, r_{0}$ and $k$ are derivative of modified Bessel function, reference radius of multipole coefficient and $2 \pi$ ddivided by the length of helical-pitch respectively. $r$ is the distance from the beam axis, and corresponds to $r_{1}$ and $r_{2}$ in Fig. 1. In data analysis only output data from the outer Hall generator, $r_{2}$, were used to calculate the dipole component. To obtain other multipole components, the values subtracting inner generator data, $r_{1}$, from outer data, $r_{2}$, were analyzed. This subtraction can eliminate bias noise. This value are expressed as

$$
\begin{aligned}
& B_{0} \sum_{n=0}^{\infty} s_{n} r_{0}\left[a_{n} \cos ((n+1) \theta)+b_{n} \sin ((n+1) \theta)\right] \cdots(3) \\
& s_{n}=\frac{f_{n}}{k(n+1)}\left[I_{n+1}^{\prime}\left((n+1) k r_{2}\right)-I_{n+1}^{\prime}\left((n+1) k r_{1}\right)\right] \cdots(4) .
\end{aligned}
$$

Here, $r_{0}, r_{1}, r_{2}$ and $k$ are $31 \mathrm{~mm}, 6.6 \mathrm{~mm}, 35.6 \mathrm{~mm}$ and $2 \pi /(2.4 \mathrm{~m})$ respectively, and $s_{n}$ are as shown in Table 1.

Table 1

\begin{tabular}{|c|c|c|c|c|c|c|c|}
\hline$n$ & 0 & 1 & 2 & 3 & 4 & 5 & 6 \\
\hline$s_{n}$ & 0.0008240 & 0.04933 & 0.1009 & 0.1592 & 0.2303 & 0.3184 & 0.4276 \\
\hline
\end{tabular}

These values correspond to factors in converting from obtained Fourier coefficients to the multipole coefficients. In the dipole analysis, 0.02627 was used instead of value indicated above, because the subtraction was not applied.

The measurement was done at $105 \mathrm{~A} / \mathrm{cable}$ and $220 \mathrm{~A} / \mathrm{cable}$. The maximum measurable field strength of these transverse Hall probes used in the measurement is $3.0 \mathrm{~T}$, and $220 \mathrm{~A} / \mathrm{cable}$ corresponds to about $3 \mathrm{~T}$.

\section{The error in the measurement}

Before the measurement, a pre-experiment by using a reference normal conducting magnet was done and the measurement system was checked. The results are shown in Table 2 . All the components except the dipole should be ideally less than 1 unit.

Table 2

\begin{tabular}{|c|c|c|c|c|c|}
\hline & Dipole & Quadrupole & Sextupole & Octupole & Decapole \\
\hline Field Strength (Gauss) & 13808 & -136.8 & 20.57 & 14.22 & 6.188 \\
\hline Ratio to Dipole (Unit, 10e-4) & & -99.0 & 14.9 & 10.3 & 4.48 \\
\hline
\end{tabular}

The maximum field of the reference dipole magnet is $1.4 \mathrm{~T}$ and the ratios of each component vary wiyh different field strength. It was difficult to compensate using these results, and this table was not considered in this analysis. We have to notice that same size errors may be contained by this analysis.

\section{Three dimensional field calculation}

In this field calculation, the space was divided into roughly 230000 nodes, and the conductors were described as 730 pieces of blocks. Required computation time is about 7 days. Assumed iron magnetic property is shown in Fig. 2. Packing factor of yoke was set to $99 \%$. Punching holes in yoke were neglected. 


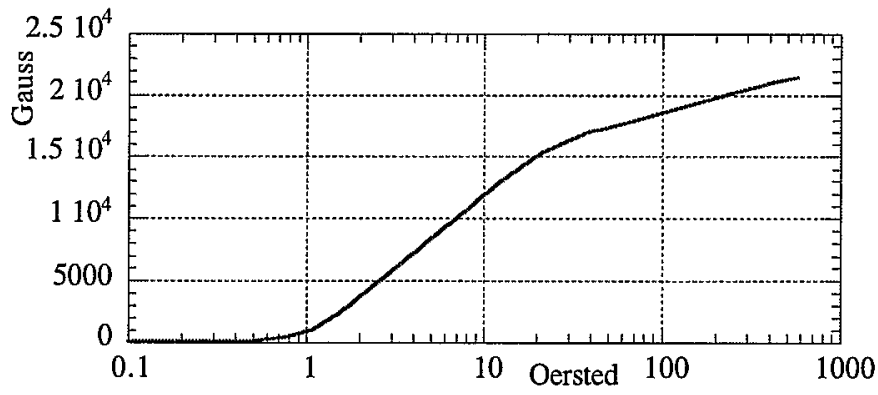

Fig. 2 B-H curve of iron yoke

\section{The comparison of measurements with calculations}

As mentioned above, currents for magnet excitation were $105 \mathrm{~A}$ and $220 \mathrm{~A}$, however prepared calculation were for $100 \mathrm{~A}$ and $200 \mathrm{~A}$. So $100 \mathrm{~A}$ calculation result was converted to for $105 \mathrm{~A}$ by multiplying 1.05 times. For $220 \mathrm{~A}$ case, the field was re-calculated after measurement. Comparisons of measurements with calculations for the dipole, sextupole and Decapole, are shown in Fig. 3 to 8 . In dipole case, agreements in both the field strength and the phase angle are very good. In the sextupole and the decapole cases, agreements in the end regions, where there are relatively strong multipole field, are excellent, however some discrepancies in the body section are observed. Yet, these discrepancies can make sense considering the errors mentioned above. In these figures, the results from the tangential rotating coil measurement are also indicated.

\section{Conclusion}

Within the accuracy of this measurement, the predicted field by TOSCA is very reliable. Also the fabrication method of the Slotted type is trustworthy. On the other hand, the method of the Hall probe measurement and the method of the analysis can be improved. We would like to discuss these issues in another report.

\section{Acknowledgment}

The author is grateful to Animesh Jain and Richard Thomas for providing experimant data and help ful suggestions, also is gratefulf to useful discussion with Toshi Tomy Tominaka, Micheal J Syphers, Erick Willen and Takeshi Katayama. 

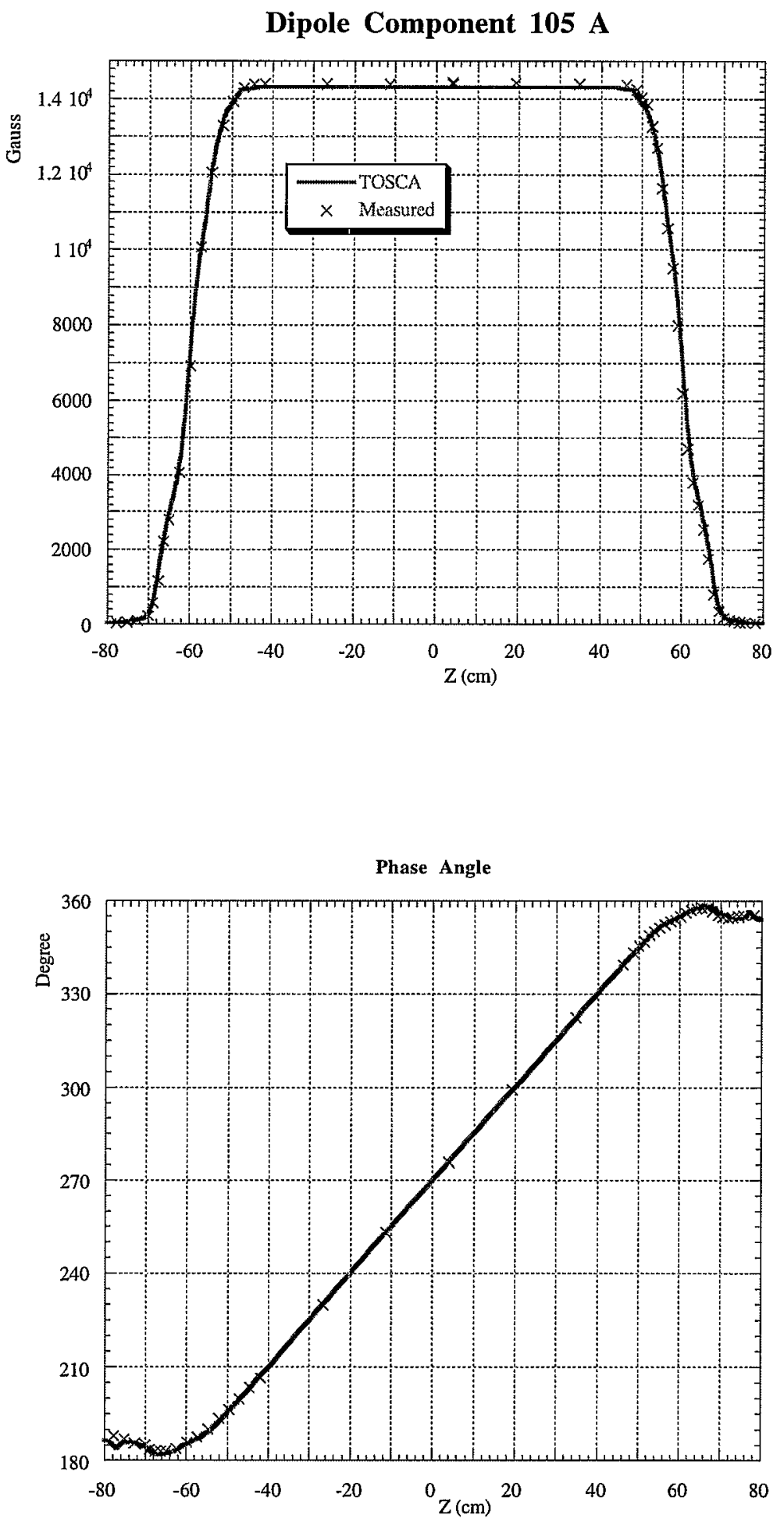

Fig. 3105 A, Dipole component 

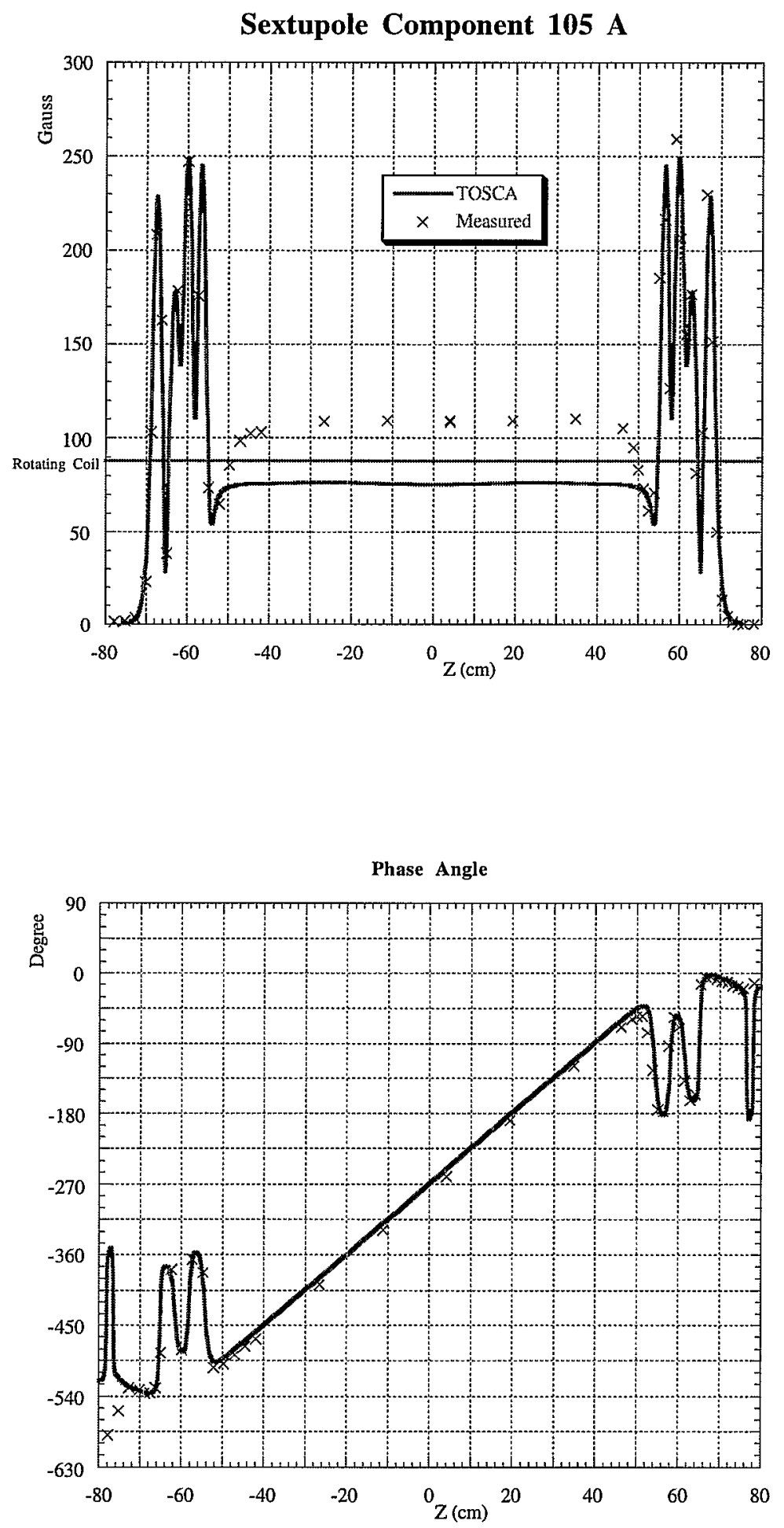

Fig. 4105 A, Sextupole component 

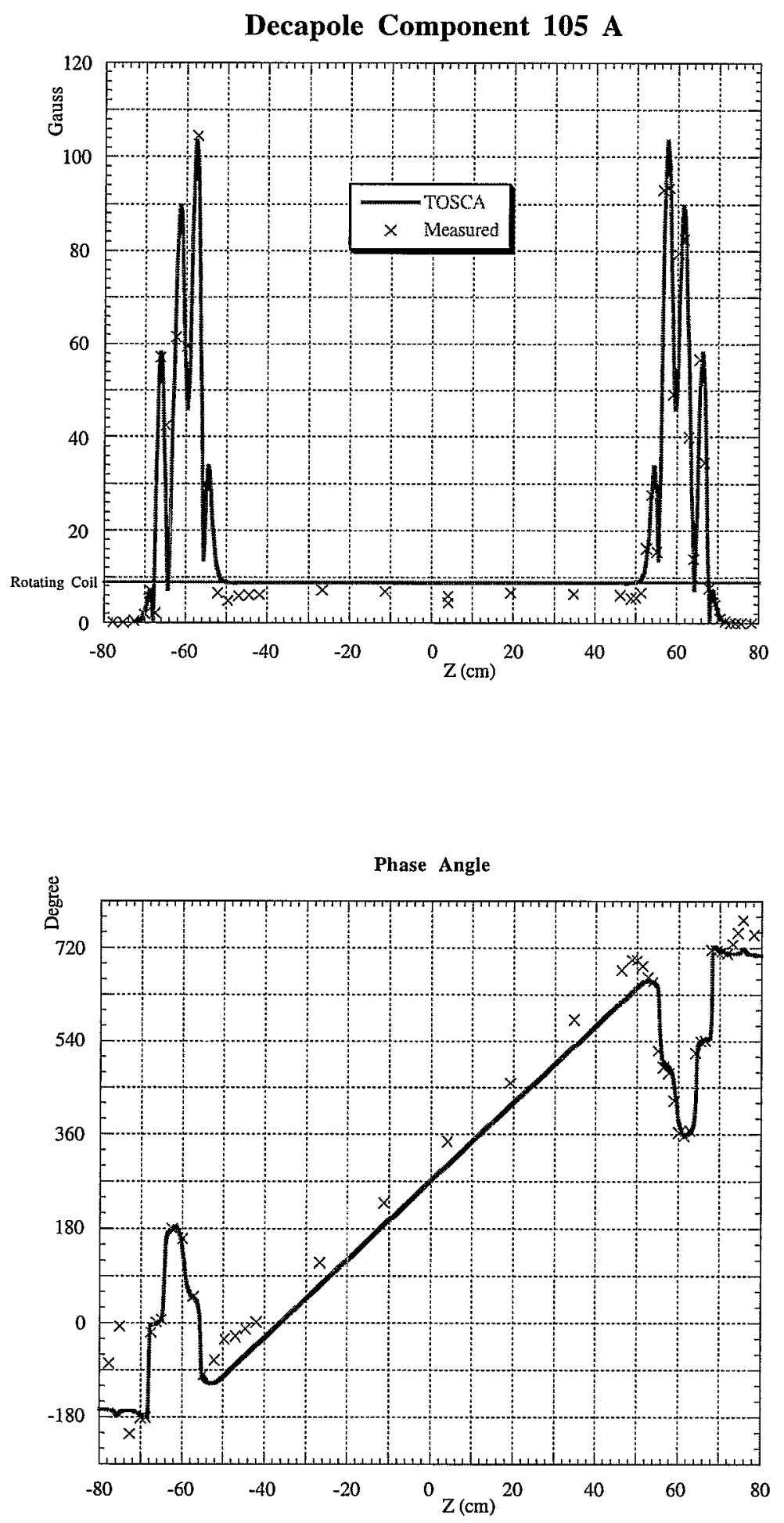

Fig. 5105 A, Decapole component 

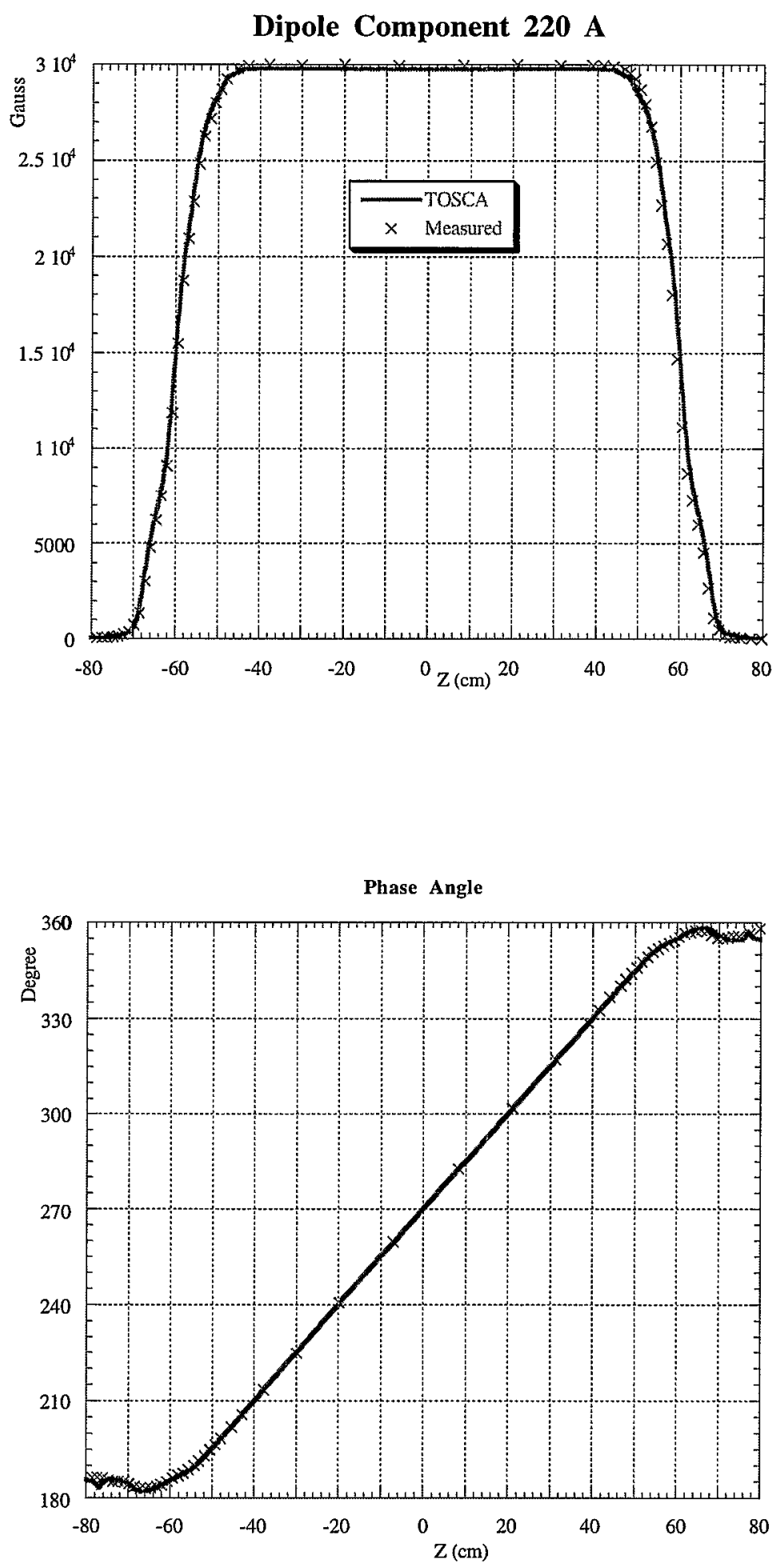

Fig. 6220 A, Dipole component 

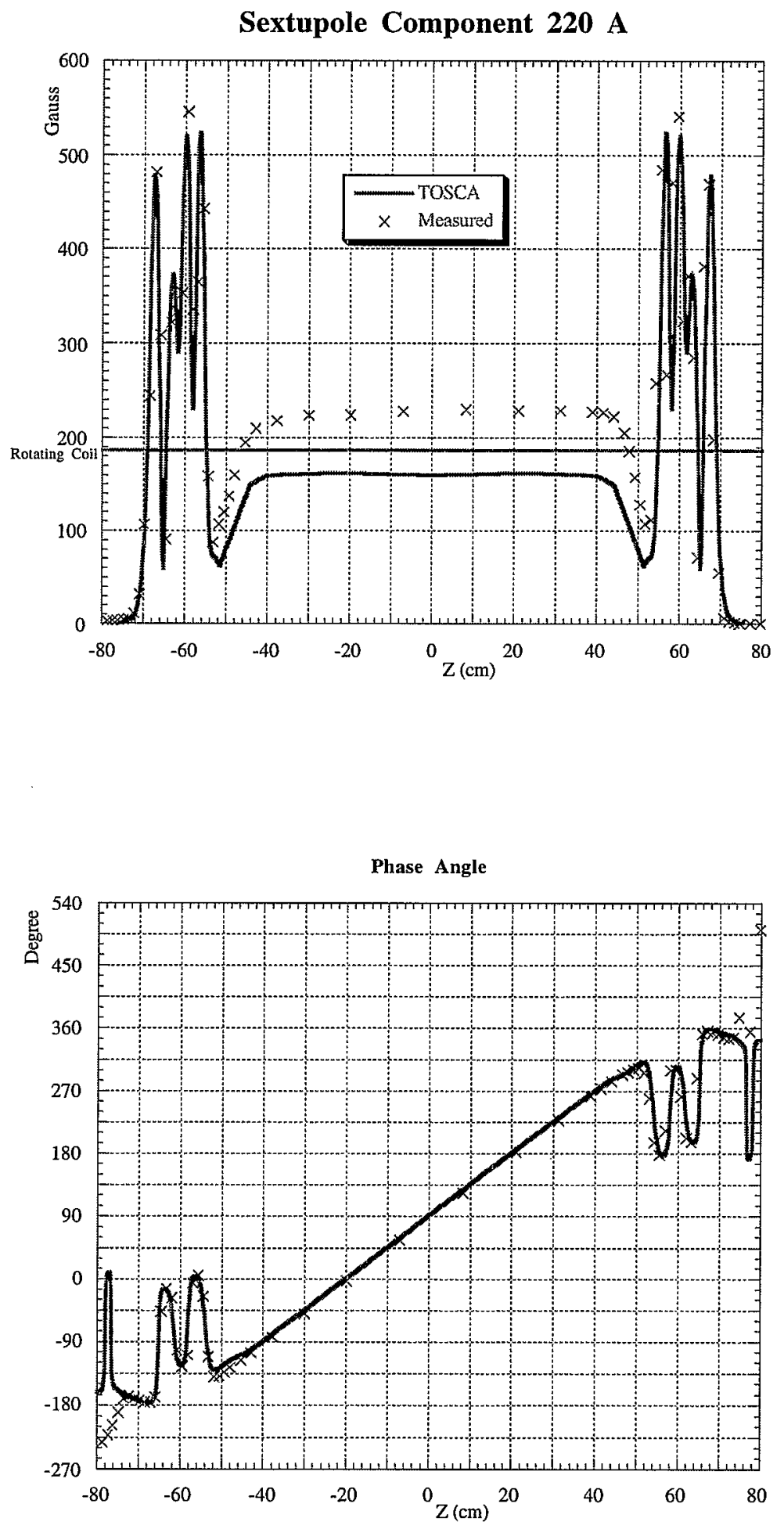

Fig. 7220 A, Sextupole component 

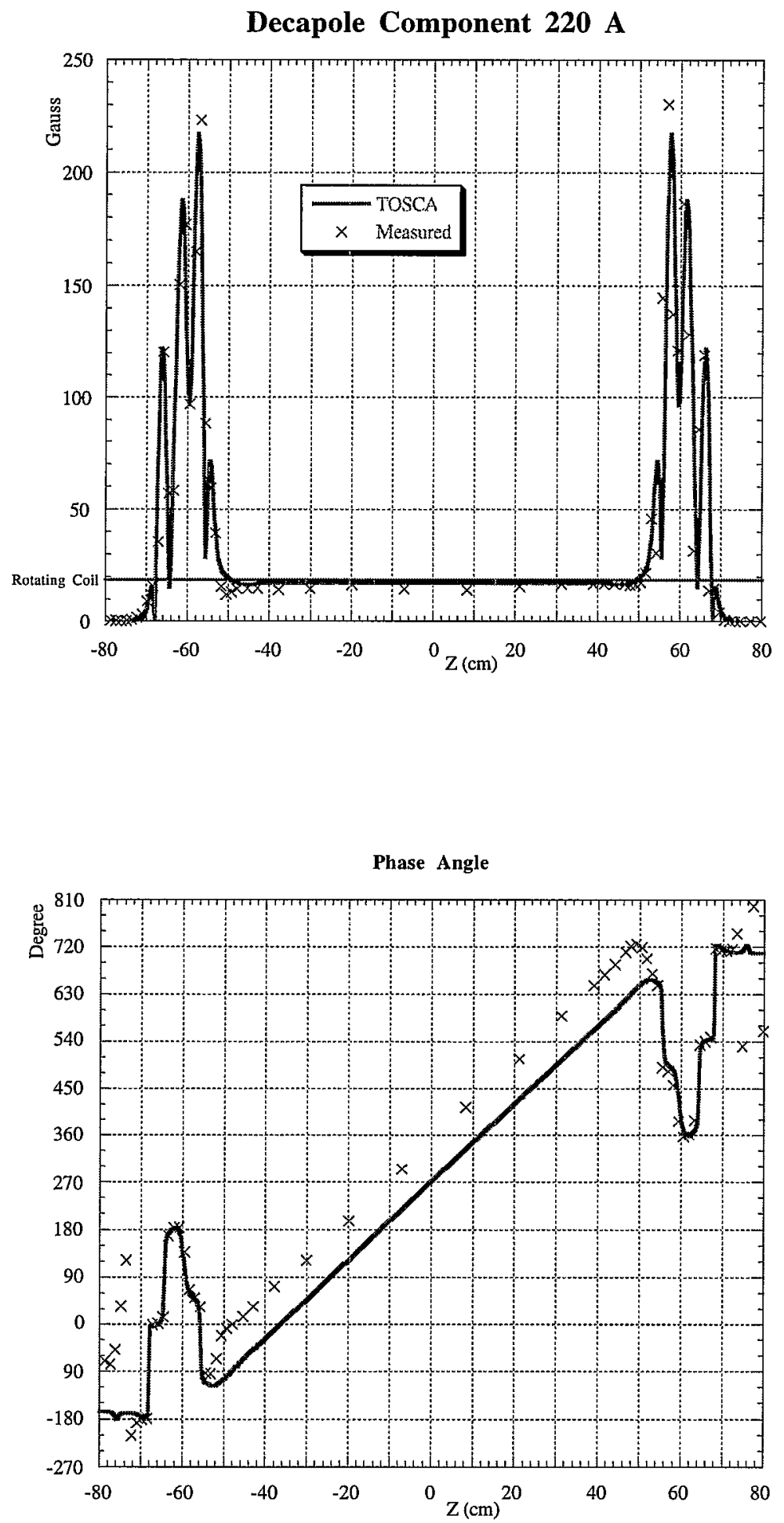

Fig. 8220 A, Decapole component 